\title{
Dynamical approach to heavy ion-induced fission
}

\author{
D.Y. Jeung ${ }^{1, \text { a }}$, E. Williams ${ }^{1}$, D.J. Hinde ${ }^{1}$, M. Dasgupta ${ }^{1}$, R. du Rietz ${ }^{1, b}$, M. Evers ${ }^{1, \text { c }}$, C.J. Lin ${ }^{1, \text { d }}$, D.H. Luong ${ }^{1}$, C. Simenel ${ }^{1}$, \\ and A. Wakhle ${ }^{1, \mathrm{e}}$ \\ ${ }^{1}$ Department of Nuclear Physics, Research School of Physics and Engineering, The Australian National University, \\ Canberra, ACT 2601, Australia
}

\begin{abstract}
Deep inelastic collisions (DICs) can compete strongly with fusion in collisions of heavy nuclei. However, standard coupled-channels calculations do not take DIC processes into account. As a result, calculations have been shown to overestimate the fusion cross-sections, resulting in a discrepancy between experimental data and theoretical calculations, particularly at energies above the fusion barrier. To investigate this discrepancy, we conducted a series of experiments using the ANU 14UD tandem accelerator and the CUBE 2-body fission spectrometer to examine the competition between transfer/DIC and fusion. In particular, fusion-fission and 3-body fission yields have been extracted for ${ }^{34} \mathrm{~S}+{ }^{232} \mathrm{Th}$ and ${ }^{40} \mathrm{Ca}+{ }^{232} \mathrm{Th}$ systems. This work shows that the transfer-fission probability is enhanced relative to fusion-fission for ${ }^{40} \mathrm{Ca}+{ }^{232} \mathrm{Th}$, when compared to ${ }^{34} \mathrm{~S}+{ }^{232} \mathrm{Th}$. It is suggested that the enhancement of this DIC process in ${ }^{40} \mathrm{Ca}+{ }^{232} \mathrm{Th}$ is linked to an increase in the density overlap of the colliding nuclei as a function of the charge product and contributes to fusion hindrance.
\end{abstract}

\section{Introduction}

Deep inelastic collisions (DICs) have been shown to compete strongly with fusion [1] and become increasingly probable as the charge product $\left(\mathrm{Z}_{1} \mathrm{Z}_{2}\right)$ of the reaction increases [2-4]. However, the quantitative role these processes play in reaction outcomes is not well understood. Therefore, understanding the DIC process is crucial for understanding fusion dynamics in heavy ion collisions.

The standard coupled-channels (CC) model used for describing the fusion process has been successful in explaining phenomena like the enhancement of fusion cross-sections at energies below the average fusion barrier [5-6]. However, the observation of fusion suppression, particularly at above-barrier energies, cannot be described by these models [7-8]. This disagreement between $\mathrm{CC}$ models and observation is thought to be linked to the contribution of DICs [7,9], which are not included within the CC framework. In Ref. [7], it was suggested that an enhancement in DICs with increasing $\mathrm{Z}_{1} \mathrm{Z}_{2}$ may be responsible for fusion suppression. Thus, a new approach that includes both $\mathrm{CC}$ effects and energy dissipation that results in DICs is required to illustrate any observable outcomes resulting from the onset of DICs. Such a dynamical reaction model is being developed at the Australian National University (ANU); the work presented here is aimed at providing experimental inputs to this phenomenological model.

One factor that is considered to be important in shaping the influence of dissipative processes on reaction outcomes is the density overlap of colliding nuclei, i.e., increasing nucleon-nucleon interactions that result in a substantial loss of kinetic energy and angular momentum from the relative motion. The relative kinetic energy of the colliding nuclei is dissipated into excitation energy (heating) of the heavy product, which can be formed by either transfer or fusion reactions. The transfer probabilities, in particular, are significantly affected by this density overlap. However, systematic experimental study of the interplay between density overlap and transfer probabilities have not been carried out, although recent experiments are addressing this issue [10-11].

\footnotetext{
${ }^{\mathrm{a}}$ D. Y. Jeung: dongyun.jeung@anu.edu.au

${ }^{\mathrm{b}}$ Current address: Malmö University, Faculty of Technology and Society, 20506 Malmö, Sweden

${ }^{c}$ Current address: Institute of Functional Genomics, University of Regensburg, 93053, Germany

${ }^{\mathrm{d}}$ Current address: China Institute of Atomic Energy, Beijing 102413, China

${ }^{\mathrm{e}}$ Current address: National Superconducting Cyclotron Laboratory, Michigan State University, East Lansing, Michigan 48824, USA
} 
One way of examine the influence of dissipative processes is to study fusion-fission (or called as full momentum transfer (FMT) fission) and transfer-fission reactions. FMT fission corresponds to fission that occurs when a projectile has been completely absorbed into a target and formed a thermally equilibrated composite nucleus before undergoing fission. In contrast, transferfission corresponds to fission from a target-like (heavy) nucleus, formed following nucleon exchange between colliding nuclei, that has an excitation energy higher than the fission barrier.

In this paper, FMT fission and transfer-fission yields have been extracted for ${ }^{34} \mathrm{~S}+{ }^{232} \mathrm{Th}$ and ${ }^{40} \mathrm{Ca}+{ }^{232} \mathrm{Th}$ reactions. The comparison between these reactions will shed light on the effect of the DIC process on heavy ion collisions and explore the necessity of explicitly taking energy dissipation into account in the description of the fusion process.

\section{Experimental Details}

The experiments were carried out at the Heavy Ion Accelerator Facility at the ANU. The 14UD electrostatic accelerator was used to produce a pulsed beam of $\sim 1.3 \mathrm{~ns}$ width at intervals of $\sim 106 \mathrm{~ns}$. For the ${ }^{40} \mathrm{Ca}+{ }^{232} \mathrm{Th}$ reaction, the ANU superconducting linear postaccelerator was used to increase the beam energies above the fusion barrier. Table 1 provides target details and energy ranges for the reactions studied.

Table 1. Reaction parameters.

\begin{tabular}{|c|c|c|c|}
\hline Reaction & $\begin{array}{c}\text { Target } \\
\text { Thickness } \\
\left(\boldsymbol{\mu g} / \mathbf{c m}^{2}\right)\end{array}$ & $\begin{array}{c}\text { Backing, } \\
\text { Thickness } \\
\left(\boldsymbol{\mu g} / \mathbf{c m}^{2}\right)\end{array}$ & $\begin{array}{c}\text { Beam } \\
\text { Energy } \\
\text { Ranges } \\
(\mathbf{M e V})\end{array}$ \\
\hline${ }^{34} \mathrm{~S}+{ }^{232} \mathrm{Th}$ & 80 & $\mathrm{C}, 10$ & $155-192$ \\
\hline${ }^{40} \mathrm{Ca}+{ }^{232} \mathrm{Th}$ & 80 or 330 & $\mathrm{C}, 10$ or $\mathrm{Al}, 30$ & $213-264$ \\
\hline
\end{tabular}

Coincident fission fragments were detected in the CUBE detector, which consists of two large-area position-sensitive multi-wire proportional counters (MWPCs) [12]. A schematic view of the experimental setup is given in Figure 1.

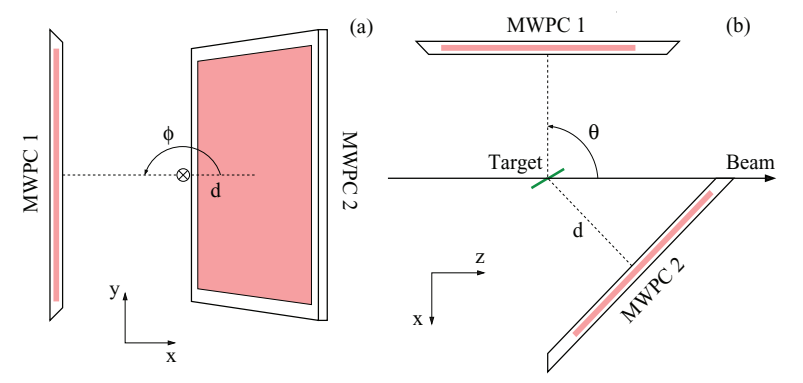

Figure 1. Experimental setup of the CUBE binary fission spectrometer. Labels 1 and 2 correspond to the back and front detectors respectively; (a) shows the azimuthal positioning where the beam is going into the page, and (b) shows the configuration as seen from above. The position of detectors is defined by their distance from the target (d), azimuthal angle $(\phi)$ and polar angle $(\theta)$ relative to the beam axis [12].

The two detectors were placed $180 \mathrm{~mm}$ away from the target. The front detector was centred at $\theta_{2}=45^{\circ}$, providing an angular coverage from $5^{\circ}$ to $80^{\circ}$. We used two different ranges of angular coverage for the back detector. It was centred at $\theta_{1}=90^{\circ}$ with either $55^{\circ}$ to $130^{\circ}$ or $50^{\circ}$ to $125^{\circ}$ coverage, depending on the orientation of the back detector. $\mathrm{X}$ and $\mathrm{Y}$ anode planes, made up of a grid of $20 \mu \mathrm{m}$ gold plated tungsten wires, provided position information, and the centre foil provided timing and energy loss information.

The coincident detection of fragments allowed the determination of the fission fragment mass ratio and velocities through kinematic reconstruction based on twobody kinematics [13].

\section{Data Analysis}

The data analysis procedure is largely divided into three steps. Each step of the analysis is explained below, using the ${ }^{40} \mathrm{Ca}+{ }^{232} \mathrm{Th}$ reaction at $E_{\text {lab }}=263.3 \mathrm{MeV}$ as an example.

\subsection{Selecting the Fission Events}

There are two types of data that need to be eliminated in order to select the fission events clearly; (1) those corresponding to elastically scattered particles and (2) coincident events occurring due to reactions with lighter elements in the target and detection of one fission fragment in coincidence with a beam-like particle.

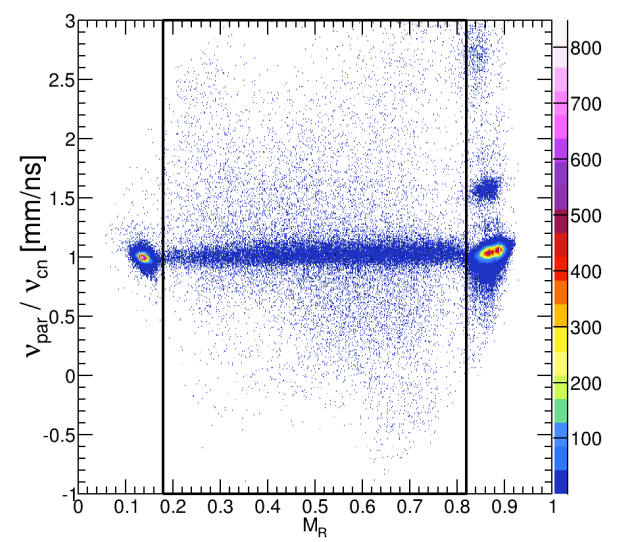

Figure 2. Scatter plot of mass ratio $\mathrm{M}_{\mathrm{R}}$ vs $v_{\mathrm{par}} / v_{c n}$. The scale of counts is given by a colour scheme in the right panel. The rectangular gate (black lines) is used to select fission events and remove the elastically scattered events.

The parallel velocity component $\left(v_{p a r}\right)$ for the elastic scattering events is the same as the compound nuclear velocity $\left(v_{c n}\right)$ and thus these events can overlap with the fusion-fission events. The elastically scattered events were clearly identified in the mass ratio plot, $M_{R}$ vs 
$v_{\text {par }} / v_{c n}$, as the two high intensity regions near $\mathrm{M}_{\mathrm{R}} \sim 0.15$ and $\mathrm{M}_{\mathrm{R}} \sim 0.85$ shown in Figure 2. To simplify the next step in the analysis, these events were eliminated using a rectangular gate as shown.
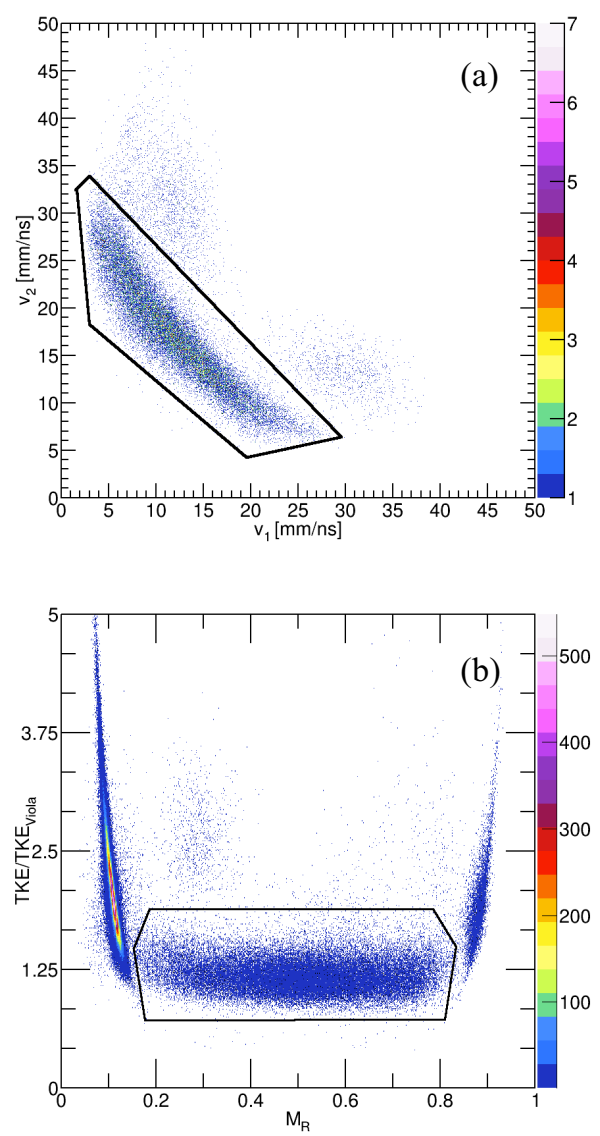

Figure 3. A polygonal gate is used to remove coincidences between beam-like nuclei and one fission fragment. (a) Scatter plot of $\mathrm{v}_{1} \mathrm{vs}_{2}$ for ${ }^{40} \mathrm{Ca}+{ }^{232} \mathrm{Th}$ reaction at $E_{\text {lab }}=263.3 \mathrm{MeV}$. (b) Scatter plot of $\mathrm{M}_{\mathrm{R}}$ vs $\mathrm{TKE} / \mathrm{TKE}_{\text {Viola }}$ for ${ }^{34} \mathrm{~S}+{ }^{232} \mathrm{Th}$ reaction at $E_{l a b}=182 \mathrm{MeV}$. The scale of counts is given by a colour scheme in the right panel.

In addition to the elastics, events due to random coincidences between beam-like nuclei and one fission fragment can be present. These were separated by putting an additional gate in the $v_{1}-v_{2}$ plot as shown in Figure 3 (a).

For the ${ }^{34} \mathrm{~S}+{ }^{232} \mathrm{Th}$ reaction analysis, an additional polygonal gate was applied to the scatter plot of $M_{R}$ versus relative total kinetic energy (RTKE) in order to further exclude unwanted events. This gate is shown in Figure 3 (b). RTKE is defined as $T K E_{\text {exp }} / T K E_{\text {Viola }}$ where $T K E_{\text {exp }}$ is the total kinetic energy determined from the experimental data and $T K E_{\text {Viola }}$ is the kinetic energy derived from the Viola systematics [14].

\subsection{Angular Acceptance Ranges}

In addition to fusion-fission, transfer-fission becomes an important process for heavier projectile and target systems. The CUBE detector can efficiently detect the fusion-fission events for ${ }^{34} \mathrm{~S}+{ }^{232} \mathrm{Th}$ and ${ }^{40} \mathrm{Ca}+{ }^{232} \mathrm{Th}$ systems but can lose events following transfer fission. This is because as the projectile becomes heavier, a larger momentum is transferred to the target, leading to a larger recoil velocity. The fragments following the fission of a composite systems formed in such reactions will have a folding angle that goes beyond the CUBE detector geometry, meaning that only one of the fragments is detected. To make a direct comparison between fusionfission and transfer-fission yields, a careful selection of the detector angles is required in the analysis to avoid missing such 3-body events.

To ensure that we had adequate solid angle coverage for both fusion-fission and transfer-fission, we looked at the position distribution of fission fragments in the front detector (see Figure 4 (b)) for different gates in the back detector angle plot (see Figure 4 (a)). Figure 4 (b) is an example of fission fragments following transfer reactions detected in the front detector after restricting the back detector angular coverage. The detail of selecting transfer-fission events is explained in the following section. If the fission fragments lie close to the black line boundary that corresponds to the edge of the front detector angular coverage, some of fission fragments might be missed. Therefore, we systematically searched for the back detector (1) position gates that kept fission fragments away from the front detector (2) edges.
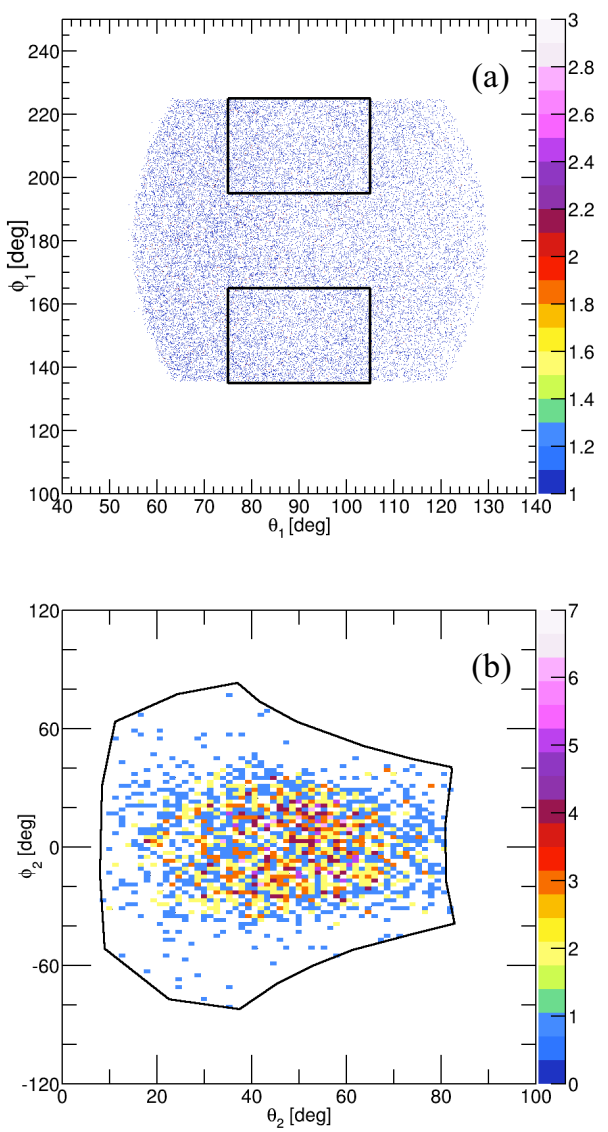

Figure 4. Scatter plot of fission fragments observed in the back (a) and front (b) detectors for the ${ }^{40} \mathrm{Ca}+{ }^{232} \mathrm{Th}$ reaction at $E_{\text {lab }}=$ 263.3 MeV. The scale of counts is given by a colour scheme in 
the right panel. (a) The top rectangular box represents fission fragments having negative velocities while the bottom rectangular box represents those having positive velocities. (b) Black line is a boundary of the front detector angular coverage. The events at the centre of the plot in (b) correspond to pairs of fission fragments caught following a transfer-fission reaction.

Applying these gates in (a) gives the fission fragments from the transfer-fission seen in (b).

In the ${ }^{34} \mathrm{~S}+{ }^{232} \mathrm{Th}$ reactions, pairs of the fission fragments could be detected efficiently in both detectors by defining one rectangular gate between $75^{\circ}<\theta_{1}<105^{\circ}$ and $157.5^{\circ}$ $<\phi_{1}<202.5^{\circ}$ in the back detector and looking for the complimentary fission fragment in the front detector, ensuring fission fragment events were clustered away from the edges.

However, the above method did not give efficient detection of the fission pairs for the ${ }^{40} \mathrm{Ca}+{ }^{232} \mathrm{Th}$ system. Hence, two sets of $\theta-\phi$ gates were put on the back detector. In the first case, a gate including the angular ranges $75^{\circ}<\theta_{1}<105^{\circ}$ and $135^{\circ}<\phi_{1}<165^{\circ}$ was put on the back detector: this corresponded to positive perpendicular velocity of the fission pair. In the second, for the same angular coverage in $\theta_{1}, 195^{\circ}<\phi_{1}<225^{\circ}$ was selected. This gate corresponded to negative velocity of the fission pair. The total fission events were the sum of fission pairs extracted respectively from the two sets of gates shown in Figure 4 (a).

\subsection{Separation of Fusion-fission and Transfer- fission}

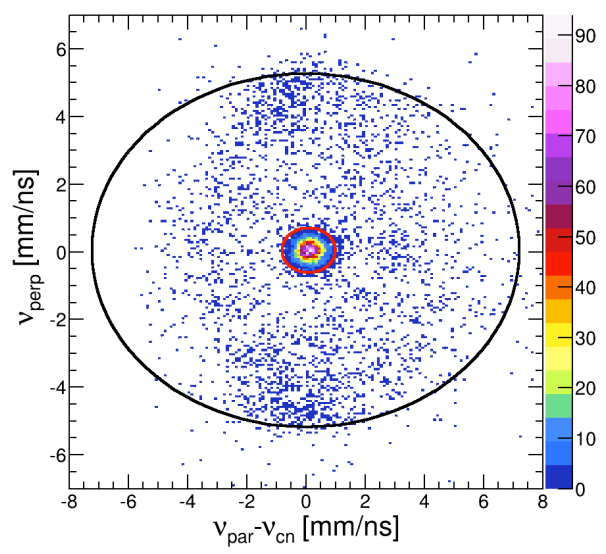

Figure 5. Fission fragment velocity components parallel and perpendicular to the beam. Fusion-fission events lie in the centre of the figure (red line), while transfer-fission events are spread out within an ellipse (black line). The scale of counts is given by a colour scheme in the right panel.

Figure 5 shows a scatter plot of the measured $v_{p a r}-v_{c n}$ against $v_{\text {perp }}$, where $v_{\text {perp }}$ is the velocity component perpendicular to the beam axis. For fission following a fusion reaction, fission fragments travel with the same centre-of-mass velocity as the parallel component of the compound nucleus velocity and zero perpendicular velocity. A small spread in fusion-fission events results from the evaporation of light particles such as $n, p, \alpha$ particles from the nuclei either before or after scission, as well as the position/time resolution of the detector. Therefore, the high intensity region in the centre of the plot corresponds to fusion-fission. This region is bounded within an elliptical shape. The lengths of the major and minor axes of the ellipse were defined from the widths of a 1-D Gaussian fit to the projection of the $v_{p a r}-v_{c n}$ versus $v_{\text {perp }}$ plot.

Outside this region, the 3-body events corresponding to fission following a transfer reaction are located. These events are associated with reactions resulting in a projectile-like nucleus and two fission fragments. These fragments are constrained by kinematic limits, and thus they are spread inside a circle of radius $v_{c n}$, centred at $v_{p a r}{ }^{-}$ $v_{c n},=0 v_{\text {perp }}=0$. However, some of these events cross the kinematic limits due to some instrumental effects or resolution issues. Therefore, an elliptical shape boundary was used for selecting transfer-fission events.

\section{Results and Discussion}

This paper focuses on studying the ratio of 3-body events (comprising one projectile-like nucleus and two fission fragments arising from fission of the target-like nucleus) to FMT fission following capture. For simplicity, we denote this as the ratio of transfer-fission to fusionfission.

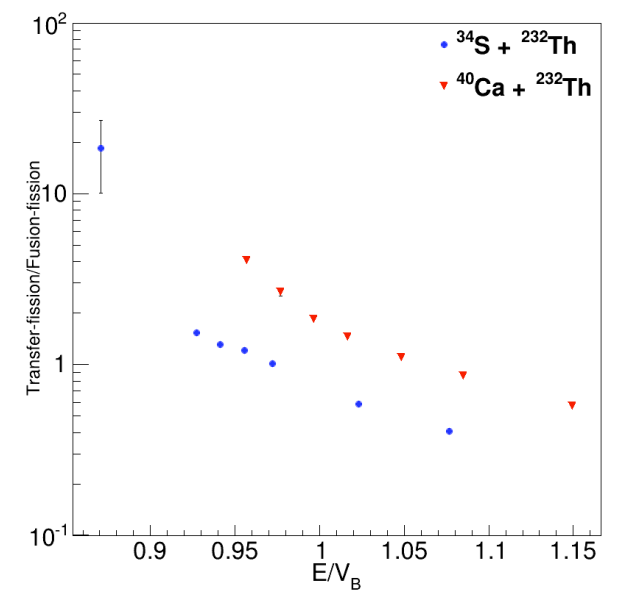

Figure 6. Ratio of transfer-fission to fusion-fission with respect to the reduced energy.

Figure 6 shows the ratio of transfer-fission to fusionfission events as a function of the beam energy normalised by the proximity barrier $V_{B}$ [15]. The errors on the ratios were obtained from the error propagation of the statistical errors.

As Figure 6 shows, for reactions involving heavy projectiles, a higher ratio is observed as compared to reactions involving light projectiles for the full range of measured energies.

The results show that with increasing $Z_{1} Z_{2}$, transferfission is more probable than fusion-fission process. This is consistent with the idea that an increasing density 
overlap between the colliding nuclei results in an increase in transfer reactions, which in turn competes with fusion reactions. Thus, an increase in the transfer probability leads to an increase in fusion suppression due to loss of flux from the fusion channel. This result is consistent with Ref. [7], which also describes an increased fusion suppression in reactions between heavier nuclei in comparison to those involving light nuclei.

\section{Conclusions}

Transfer-fission events have been well separated from fusion-fission events for ${ }^{34} \mathrm{~S}+{ }^{232} \mathrm{Th}$ and ${ }^{40} \mathrm{Ca}+{ }^{232} \mathrm{Th}$ reactions using the kinematic coincidence method. The measurements show that transfer-fission reactions increase with an increase in $\mathrm{Z}_{1} \mathrm{Z}_{2}$ relative to fusionfission. This relative increase in transfer with the charge product may be linked to an enhancement in the density overlap for collisions between heavier nuclei. This observation provides important evidence supporting the idea that DICs should be taken into account in models describing the fusion process in heavy-ion reactions.

\section{Acknowledgments}

The authors are grateful for the contribution of D. C. Weisser, N. Lobanov, T. Kibedi, and all the accelerator staff at the ANU accelerator facility. This work is supported by the Australian Research Council Grants No. FL110100098, FT120100760, DP130101569, DP140101337, DE140100784.

\section{References}

1. E. L. H. Wolfs, Phys. Rev. C 36, 1379 (1987)

2. W. J. Swiatecki, Phys. Scr. 24, 113 (1981)

3. S. Bjornholm and W. J. Swiatecki, Nucl. Phys. A 391, 471 (1982)

4. J. P. Blocki, H. Feldmeier, and W. J. Swiatecki, Nucl. Phys. A 459, 145 (1986)

5. M. Beckerman, Rep. Prog. Phys 51, 1047 (1988)

6. W. Reisdorf, J. Phys. G: Nucl. Part. Phys. 20, 1297 (1994)

7. J. O. Newton, R. D. Butt, M. Dasgupta, D. J. Hinde, I. I. Gontchar, and C. R. Morton, Phys. Rev. C 70, 024605 (2004)

8. D. J. Hinde, M. Dasgupta, and A. Mukherjee, Phys. Rev. Lett. 89, 282701 (2002)

9. J. G. Keller, B. B. Back, B. G. Glagola, D. Henderson, S. B. Kaufman, S. J. Sanders, R. H. Siemssen, F. Videbaek, B. D. Wilkins, and A. Worsham, Phys. Rev. C 36, 1364 (1987)

10. M. Evers, M. Dasgupta, D. J. Hinde, D. H. Luong, R. Rafiei, and R. du Rietz, Phys. Rev. C 84, 054614 (2011)

11. L. Corradi, G. Pollarolo, and S. Szilner, J. Phys. G 36, 113101 (2009)

12. R. du Rietz, E. Williams, D. J. Hinde, M. Dasgupta, M. Evers, C. J. Lin, D. H. Luong, C. Simenel, and A. Wakhle, Phys. Rev. C 88, 054618 (2013)
13. D. J. Hinde, M. Dasgupta, J. R. Leigh, J. C. Mein, C. R. Morton, J. O. Newton, and H. Timmers, Phys. Rev. C 53, 1290 (1996)

14. V. E. Viola, K. Kwiatkowski, and M. Walker, Phys. Rev. C 31, 1550 (1985)

15. W. J. Świątecki, K. Siwek -Wilczyńska, and J. Wilczyński, Phys. Rev. C 71, 04602 (2005) 